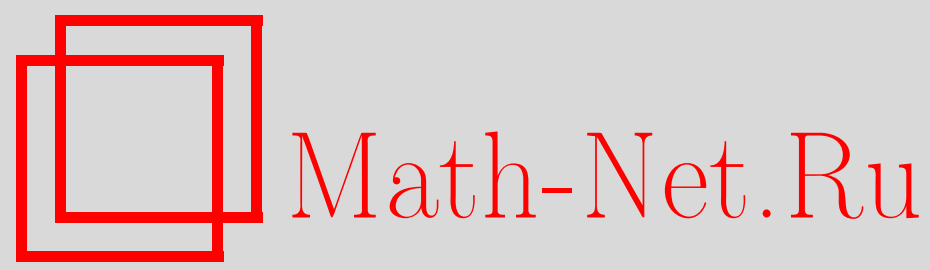

И. М. Гусейнов, Об одном операторе преобразования, $M a$ тем. заметки, 1997, том 62, выпуск 2, 206-215

DOI: https://doi.org/10.4213/mzm1605

Использование Общероссийского математического портала Math-Net.Ru подразумевает, что вы прочитали и согласны с пользовательским соглашением http://www .mathnet.ru/rus/agreement

Параметры загрузки:

IP: 35.173.219.149

26 апреля 2023 г., 18:22:46

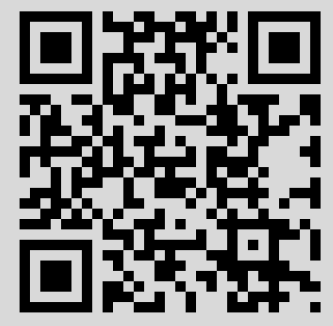


уДК 517.9

\section{ОБ ОДНОМ ОПЕРАТОРЕ ПРЕОБРАЗОВАНИЯ}

\section{И.М. Гусейнов}

Доказьвается существование оператора преобразования с условием на бесконечности, который переводит решение уравнения $y^{\prime \prime}=\lambda^{2 n} y$ в решение уравнения

$$
y^{\prime \prime}-\left(q_{0}(x)+\lambda q_{1}(x)+\cdots+\lambda^{n-1} q_{n-1}(x)\right) y=\lambda^{2 n} y .
$$

Изучаются некоторые свойства ядра оператора преобразования.

Библиографоия: 10 названий.

Рассмотрим дифференциальное уравнение

$$
y^{\prime \prime}-\sum_{k=0}^{n-1} \lambda^{k} q_{k}(x) y=\lambda^{2 n} y
$$

на полуоси $0 \leqslant x<\infty$, где $\lambda$ - комплексньй параметр; $y=y(x, \lambda)$ - неизвестная функция. Предположим, что выполняются следующие условия:

а) функции $q_{1}(x), \ldots, q_{n-1}(x)$ диффференцируемы на полуоси $(0, \infty)$;

б) интегралы

$$
\int_{0}^{\infty}(1+u)^{1-1 / n}\left|q_{k}^{\prime}(u)\right| d u, \quad k=1,2, \ldots, n-1,
$$

сходятся, и $\sigma(0)<+\infty$, где

$$
\sigma(x)=\sum_{k=0}^{n-1} \frac{2^{1-k / n}}{\Gamma(2-k / n)} \int_{x}^{\infty}(1+u)^{1-k / n}\left|q_{k}(u)\right| d u
$$

$\Gamma(\cdot)$ - гамма-функция Эйлера.

В данной работе доказывается, что при условиях а), б) существует оператор преобразования (общее определение таких операторов имеется в [1], [2]), которьй переводит решение $\exp \left\{-\lambda^{n} x\right\}$ простого уравнения $y^{\prime \prime}=\lambda^{2 n} y$ в решение уравнения (1) с условием на бесконечности. Заметим, что при $n=1$ оператор преобразования полностью изучен в работах [3], [4]. Поэтому в дальнейшем будем считать $n>1$. Оказывается, в этом случае для исследования свойств ядра оператора преобразования естественно использовать интегралы и производные Римана-Лиувилля дробного порядка. 
Для формулировки основного результата работы введем следующие обозначения:

$$
\begin{gathered}
\sigma_{1}(x)=\int_{x}^{\infty}\left|q_{0}(u)\right| d u+\sum_{k=1}^{n-1} \frac{2^{1-k / n}}{\Gamma(2-k / n)} \int_{x}^{\infty}(1+u)^{1-k / n}\left|q_{k}^{\prime}(u)\right| d u \\
\|\varphi\|(x)=\int_{0}^{\infty}|\varphi(x, t)| d t, \quad D_{x}=\frac{\partial}{\partial x} .
\end{gathered}
$$

Через $I_{t}^{\alpha}$ и $D_{t}^{\alpha}(0<\alpha<1)$ обозначим соответственно операторы интегрирования и дифференцирования дробного порядка по $t$ (см. [5], [6]):

$$
I_{t}^{\alpha} \varphi(x, t)=\frac{1}{\Gamma(\alpha)} \int_{0}^{t}(t-s)^{\alpha-1} \varphi(x, s) d s, \quad D_{t}^{\alpha} \varphi(x, t)=\frac{\partial}{\partial t} I_{t}^{1-\alpha} \varphi(x, t) .
$$

ТеоремА. При условиях а) и б) уравнение (1) для всех $\lambda$ из замкнутого множества $\Delta_{0}=\{\lambda:|\arg \lambda| \leqslant \pi /(2 n)\}$ имеет решение $f(x, \lambda)$, удовлетворяющее условию

$$
\lim _{x \rightarrow \infty} f(x, \lambda) \exp \left\{\lambda^{n} x\right\}=1,
$$

и существует ядро $A(x, t)$ оператора преобразования такое, что

$$
f(x, \lambda)=\exp \left\{-\lambda^{n} x\right\}+\int_{x}^{\infty} A(x, t-x) \exp \left\{-\lambda^{n} t\right\} d t .
$$

Ядро $A(x, t)$ обладает следующими свойствами:

1) выполнено

$$
\|A\|(x) \leqslant \exp \{\sigma(x)\}-1
$$

2) на полуоси $(0, \infty)$ существуют суммируемые по $t$ производные $D_{x} A(x, t)$, $D_{t}^{1 / n} A(x, t), \ldots,\left(D_{t}^{1 / n}\right)^{n} A(x, t)$, причем

$$
\left\|D_{x} A\right\|(x) \leqslant \sigma_{1}(x)(1+\sigma(x)) \exp \{\sigma(x)\} ;
$$

3) выполнено

$$
\begin{gathered}
\left.I_{t}^{(n-1) / n}\left(D_{t}^{1 / n}\right)^{m} A(x, t)\right|_{t=0}=\sum_{k=0}^{m} \frac{1}{(m-k+1) !}\left(\frac{1}{2} \int_{x}^{\infty} q_{n-k-1}(u) d u\right)^{m-k+1} \\
\lim _{t \rightarrow \infty} I_{t}^{(n-1) / n}\left(D_{t}^{1 / n}\right)^{m} A(x, t)=0, \quad m=0,1, \ldots, n-1
\end{gathered}
$$

4) если функиии $q_{0}(x), q_{1}^{\prime}(x), \ldots, q_{n-1}^{\prime}(x)$ имеют непрерывные производные и интеграль

$$
\int_{0}^{\infty}(1+u)\left|q_{0}^{\prime}(u)\right| d u, \quad \int_{0}^{\infty}(1+u)^{1-1 / n}\left|q_{k}^{\prime \prime}(u)\right| d u, \quad k=1,2, \ldots, n-1,
$$

сходятся, то функиия $A(x, t)$ удовлетворяет уравнению

$$
D_{x}^{2} A(x, t)-2 D_{x}\left(D_{t}^{1 / n}\right)^{n} A(x, t)-\sum_{k=0}^{n-1} q_{k}(x)\left(D_{t}^{1 / n}\right)^{k} A(x, t)=0 .
$$

Предварительно докажем одно вспомогательное утверждение, представляющее также самостоятельньй интерес. 
ЛЕмма. Если функиия $q(t)$ дифферениируема на $(0, \infty)$ u

$$
\int_{0}^{\infty}(1+t)^{1-\alpha}\left|q^{\prime}(t)\right| d t<+\infty, \quad 0<\alpha<1
$$

то производные $\left(D_{t}^{\alpha}\right)^{m} q(t), m=1,2, \ldots,[1 / \alpha]$, суммируемы и имеет место неравенство

$$
\int_{0}^{\infty}\left|\left(D_{t}^{\alpha}\right)^{m} q(t)\right| d t \leqslant \frac{2}{\Gamma(2-m \alpha)} \int_{0}^{\infty} t^{1-\alpha m}\left|q^{\prime}(t)\right| d t
$$

ДокАЗАТЕЛЬСТво. Из условий леммы следует, что существует производная $D_{t}^{\alpha} q(t)$, причем

$$
D_{t}^{\alpha} q(t)=\frac{q(0)}{\Gamma(1-\alpha)} t^{-\alpha}+\frac{1}{\Gamma(1-\alpha)} \int_{0}^{t}(t-s)^{-\alpha} q^{\prime}(s) d s
$$

(см. [5], [6]). Представляя это соотношение в виде

$$
D_{t}^{\alpha} q(t)=-\frac{1}{\Gamma(1-\alpha)} t^{-\alpha} \int_{t}^{\infty} q^{\prime}(s) d s+\frac{1}{\Gamma(1-\alpha)} \int_{0}^{t}\left((t-s)^{-\alpha}-t^{-\alpha}\right) q^{\prime}(s) d s,
$$

имеем

$$
\begin{aligned}
\int_{0}^{\infty}\left|D_{t}^{\alpha} q(t)\right| d t \leqslant & \frac{1}{\Gamma(1-\alpha)} \int_{0}^{\infty} t^{-\alpha} d t \int_{t}^{\infty}\left|q^{\prime}(s)\right| d s \\
& +\frac{1}{\Gamma(1-\alpha)} \int_{0}^{\infty} d t \int_{0}^{t}\left((t-s)^{-\alpha}-t^{-\alpha}\right)\left|q^{\prime}(s)\right| d s \\
= & \frac{2}{\Gamma(1-\alpha)} \int_{0}^{\infty} s^{1-\alpha}\left|q^{\prime}(s)\right| d s,
\end{aligned}
$$

т.е. для $m=1$ лемма доказана. Для остальных значений $m$ она доказывается аналогично, исходя из равенства

$$
\left(D_{t}^{\alpha}\right)^{m} q(t)=\frac{q(0)}{\Gamma(1-\alpha m)} t^{-\alpha m}+\frac{1}{\Gamma(1-\alpha m)} \int_{0}^{t}(t-s)^{-\alpha m} q^{\prime}(s) d s .
$$

СлЕДСТвиЕ. При условиях леммы имеет место оченка

$$
\int_{0}^{\infty}\left|\left(D_{t}^{\alpha}\right)^{m} q\left(x+\frac{t}{2}\right)\right| d t \leqslant \frac{2^{2-m \alpha}}{\Gamma(2-m \alpha)} \int_{x}^{\infty} t^{1-\alpha m}\left|q^{\prime}(t)\right| d t, \quad x \geqslant 0 .
$$

ДОКАЗАТЕЛЬСТВО ТЕОРЕМЫ Проведем в Несколько этаПов.

1. Сначала докажем существование решения уравнения (1), удовлетворяющее условию (2) со свойствами (3) и (4).

Рассмотрим интегральное уравнение

$$
f(x, \lambda)=\exp \left\{-\lambda^{n} x\right\}+\int_{x}^{\infty} \frac{\operatorname{sh}\left\{\lambda^{n}(t-x)\right\}}{\lambda^{n}} \sum_{k=0}^{n-1} \lambda^{k} q_{k}(t) f(t, \lambda) d t, \quad \lambda \in \Delta_{0},
$$


эквивалентное задаче (1), (2), и будем искать решение этого уравнения в виде (3). Для того чтобы функция такого вида удовлетворяла уравнению (10), должно вьполняться равенство

$$
\begin{gathered}
\int_{0}^{\infty} A(x, t) \exp \left\{-\lambda^{n} t\right\} d t=\int_{x}^{\infty} \frac{1-\exp \left\{-2 \lambda^{n}(t-x)\right\}}{2 \lambda^{n}} \sum_{k=0}^{n-1} \lambda^{k} q_{k}(t) d t \\
+\int_{x}^{\infty} \frac{1-\exp \left\{-2 \lambda^{n}(t-x)\right\}}{2 \lambda^{n}} \sum_{k=0}^{n-1} \lambda^{k} q_{k}(t) \int_{0}^{\infty} A(t, s) \exp \left\{-\lambda^{n} s\right\} d s d t
\end{gathered}
$$

и наоборот, если функция $A(x, t)$ удовлетворяет равенству $(11)$, то функция $(3)$ является решением уравнения (10), т.е. и решением задачи $(1),(2)$.

Используя формулу (см., например, [7, с. 408])

$\frac{\exp \left\{-\lambda^{n} t\right\}}{\lambda^{n-k}}=\frac{1}{\Gamma(1-k / n)} \int_{t}^{\infty}(u-t)^{-k / n} \exp \left\{-\lambda^{n} u\right\} d u, \quad \lambda \in \Delta_{0}, \quad k=1,2, \ldots, n-1$,

преобразуем правую часть равенства (11) так, чтобы она имела вид, аналогичньй левой части. Так как

$$
\begin{aligned}
\frac{1-\exp \left\{-2 \lambda^{n}(t-x)\right\}}{2 \lambda^{n-k}}= & \frac{1}{2 \Gamma(1-k / n)}\left(\int_{0}^{\infty} u^{-k / n} \exp \left\{-\lambda^{n} u\right\} d u\right. \\
& \left.-\int_{2(t-x)}^{\infty}(u-2 t+2 x)^{-k / n} \exp \left\{-\lambda^{n} u\right\} d u\right),
\end{aligned}
$$

для первого слагаемого в правой части (11) получаем

$$
\begin{gathered}
\int_{x}^{\infty} \frac{1-\exp \left\{-2 \lambda^{n}(t-x)\right\}}{2 \lambda^{n}} \sum_{k=0}^{n-1} \lambda^{k} q_{k}(t) d t=\sum_{k=0}^{n-1} \frac{1}{2 \Gamma(1-k / n)} \int_{0}^{\infty}\left(t^{-k / n} \int_{x}^{\infty} q_{k}(u) d u\right. \\
\left.-\int_{x}^{x+t / 2}(t+2 x-2 u)^{-k / n} q_{k}(u) d u\right) \exp \left\{-\lambda^{n} t\right\} d t
\end{gathered}
$$

Аналогично можно показать, что второе слагаемое в правой части равенства (11) преобразуется к виду

$$
\begin{aligned}
\int_{x}^{\infty} & \frac{1-\exp \left\{-2 \lambda^{n}(t-x)\right\}}{2 \lambda^{n}} \sum_{k=0}^{n-1} \lambda^{k} q_{k}(t) \int_{0}^{\infty} A(t, s) \exp \left\{-\lambda^{n} s\right\} d s d t \\
= & \sum_{k=0}^{n-1} \frac{1}{2 \Gamma(1-k / n)} \int_{0}^{\infty}\left(\int_{0}^{t}(t-s)^{-k / n} \int_{x}^{\infty} q_{k}(u) A(u, s) d u d s\right. \\
& \left.-\int_{0}^{t} d s \int_{x}^{x+(t-s) / 2}(t+2 x-2 u-s)^{-k / n} q_{k}(u) A(u, s) d u\right) \exp \left\{-\lambda^{n} t\right\} d t .
\end{aligned}
$$


Отсюда и из формулы (13) следует, что равенство (11) вьполняется при всех значениях $\lambda \in \Delta_{0}$, если функция $A(x, t)$ удовлетворяет интегральному уравнению

$$
\begin{aligned}
A(x, t)= & \sum_{k=0}^{n-1} \frac{1}{2 \Gamma(1-k / n)}\left(t^{-k / n} \int_{x}^{\infty} q_{k}(u) d u-\int_{x}^{x+t / 2}(t+2 x-2 u)^{-k / n} q_{k}(u) d u\right) \\
& +\sum_{k=0}^{n-1} \frac{1}{2 \Gamma(1-k / n)} \int_{0}^{t}\left((t-s)^{-k / n} \int_{x}^{\infty} q_{k}(u) A(u, s) d u\right. \\
& \left.-\int_{x}^{x+(t-s) / 2}(t+2 x-2 u-s)^{-k / n} q_{k}(u) A(u, s) d u\right) d s
\end{aligned}
$$

Таким образом, для того чтобы закончить доказательство первой части теоремы, достаточно показать, что уравнение (14) имеет решение, удовлетворяющее неравенству (4).

К уравнению (14) применяем метод последовательных приближений, положив

$$
\begin{aligned}
A_{0}(x, t)= & \sum_{k=0}^{n-1} \frac{1}{2 \Gamma(1-k / n)}\left(t^{-k / n} \int_{x+t / 2}^{\infty} q_{k}(u) d u\right. \\
& \left.-\int_{x}^{x+t / 2}\left((t+2 x-2 u)^{-k / n}-t^{-k / n}\right) q_{k}(u) d u\right), \\
A_{l}(x, t)= & \sum_{k=0}^{n-1} \frac{1}{2 \Gamma(1-k / n)} \int_{0}^{t}\left((t-s)^{-k / n} \int_{x+(t-s) / 2}^{\infty} q_{k}(u) A_{l-1}(u, s) d u\right. \\
& \left.-\int_{x}^{x+(t-s) / 2}\left((t+2 x-2 u-s)^{-k / n}-(t-s)^{-k / n}\right) q_{k}(u) A_{l-1}(u, s) d u\right) d s, \\
& \quad l=1,2, \ldots
\end{aligned}
$$

После несложных преобразований (15) имеем

$$
\left\|A_{l}\right\|(x) \leqslant \sum_{k=0}^{n-1} \frac{2^{1-k / n}}{\Gamma(2-k / n)} \int_{x}^{\infty} u^{1-k / n}\left|q_{k}(u)\right| \cdot\left\|A_{l-1}\right\|(u) d u
$$

и так как

$$
\left\|A_{0}\right\|(x) \leqslant \sum_{k=0}^{n-1} \frac{2^{1-k / n}}{\Gamma(2-k / n)} \int_{x}^{\infty} u^{1-k / n}\left|q_{k}(u)\right| d u \leqslant \sigma(x),
$$

методом математической индукции легко доказьвается, что

$$
\left\|A_{l}\right\|(x) \leqslant \frac{(\sigma(x))^{l+1}}{(l+1) !}
$$

Отсюда следует, что ряд

$$
A(x, \cdot)=\sum_{l=0}^{\infty} A_{l}(x, \cdot)
$$

сходится равномерно по $x \in[0, \infty)$ в пространстве $L_{1}(0, \infty)$, а его сумма удовлетворяет неравенству (4), являясь одновременно решением уравнения (14). 
2. Теперь докажем свойство 2) функции $A(x, t)$. Из формулы $(15)$ следует, что функции $A_{l}(x, t), l=0,1,2, \ldots$, имеют производные по $x$, причем

$$
\begin{aligned}
D_{x} A_{0}(x, t)= & \frac{1}{2} q_{0}\left(x+\frac{t}{2}\right)+\sum_{k=1}^{n-1} \frac{1}{2 \Gamma(1-k / n)}\left(t^{-k / n} \int_{x+t / 2}^{\infty} q_{k}^{\prime}(u) d u\right. \\
& \left.-\int_{x}^{x+t / 2}\left((t+2 x-2 u)^{-k / n}-t^{-k / n}\right) q_{k}^{\prime}(u) d u\right), \\
D_{x} A_{l}(x, t)= & \int_{x}^{x+t / 2} q_{0}(u) A_{l-1}(u, t-2 u+2 x) d u \\
& +\sum_{k=1}^{n-1} \frac{1}{2 \Gamma(1-k / n)} \int_{0}^{t}\left(( t - s ) ^ { - k / n } \int _ { x + ( t - s ) / 2 } ^ { \infty } \left(q_{k}^{\prime}(u) A_{l-1}(u, s)\right.\right. \\
& \left.+q_{k}(u) D_{u} A_{l-1}(u, s)\right) d u \\
& -\int_{x}^{x+t / 2}\left((t+2 x-2 u-s)^{-k / n}-(t-s)^{-k / n}\right) \\
& \left.\times\left(q_{k}^{\prime}(u) A_{l-1}(u, s)+q_{k}(u) D_{u} A_{l-1}(u, s)\right) d u\right) d s .
\end{aligned}
$$

Аналогично оценке для $A_{l}(x, t)$ имеем

$$
\begin{aligned}
\left\|D_{x} A_{0}\right\|(x) \leqslant & \int_{x}^{\infty}\left|q_{0}(u)\right| d u+\sum_{k=1}^{n-1} \frac{2^{1-k / n}}{\Gamma(2-k / n)} \int_{x}^{\infty} u^{1-k / n}\left|q_{k}^{\prime}(u)\right| d u \leqslant \sigma_{1}(x), \\
\left\|D_{x} A_{l}\right\|(x) \leqslant & \int_{x}^{\infty}\left|q_{0}(u)\right| \cdot\left\|A_{l-1}\right\|(u) d u \\
& +\sum_{k=1}^{n-1} \frac{2^{1-k / n}}{\Gamma(2-k / n)} \int_{x}^{\infty} u^{1-k / n}\left(\left|q_{k}^{\prime}(u)\right| \cdot\left\|A_{l-1}\right\|(u)\right. \\
& \left.+\left|q_{k}(u)\right| \cdot\left\|D_{u} A_{l-1}\right\|(u)\right) d u .
\end{aligned}
$$

Отсюда, учитывая оценку (16), методом математической индукции получим

$$
\left\|D_{x} A_{l}\right\|(x) \leqslant \sigma_{1}(x) \frac{(\sigma(x))^{l}}{l !}(l+1) .
$$

Следовательно, ряд (17) можно почленно дифференцировать по $x$ и имеет место оценка (5).

Чтобы доказать суммируемость производных $\left(D_{t}^{1 / n}\right)^{m} A(x, t), m=1,2, \ldots, n$, последовательно применим оператор $D_{t}^{1 / n}$ к обеим частям уравнения $(14)$ и воспользуемся тем, что

$$
\begin{gathered}
D_{t}^{1 / n}\left(\frac{t^{-k / n}}{\Gamma(1-k / n)}\right)=\frac{t^{-(k+1) / n}}{\Gamma(1-(k+1) / n)} \\
D_{t}^{1 / n}\left(\frac{1}{\Gamma(1-k / n)} \int_{x}^{x+t / 2}(t+2 x-2 u)^{-k / n} q_{k}(u) d u\right) \\
=\frac{1}{\Gamma(1-(k+1) / n)} \int_{x}^{x+t / 2}(t+2 x-2 u)^{-(k+1) / n} q_{k}(u) d u
\end{gathered}
$$


(см. [5, с. 47]). В результате получаем

$$
\begin{aligned}
& \left(D_{t}^{1 / n}\right)^{m} A(x, t)=\sum_{k=0}^{n-m-1} \frac{1}{2 \Gamma(1-(k+m) / n)}\left(t^{-(k+m) / n} \int_{x}^{\infty} q_{k}(u) d u\right. \\
& \left.\quad-\int_{x}^{x+t / 2}(t+2 x-2 u)^{-(k+m) / n} q_{k}(u) d u\right) \\
& \quad+\sum_{k=0}^{n-m-1} \frac{1}{2 \Gamma(1-(k+m) / n)} \int_{0}^{t}\left((t-s)^{-(k+m) / n} \int_{x}^{\infty} q_{k}(u) A(u, s) d u\right. \\
& \left.\quad-\int_{x}^{x+(t-s) / 2}(t+2 x-2 u-s)^{-(k+m) / n} q_{k}(u) A(u, s) d u\right) d s \\
& \quad-\frac{1}{2} \sum_{k=0}^{m-1}\left(\frac{1}{2}\left(D_{t}^{1 / n}\right)^{k} q_{n-m+k}\left(x+\frac{t}{2}\right)-\int_{x}^{\infty} q_{n-m+k}(u)\left(D_{t}^{1 / n}\right)^{k} A(u, t) d u\right. \\
& \left.-\left.\int_{x}^{x+t / 2} q_{n-m+k}(u)\left(D_{\xi}^{1 / n}\right)^{k} A(u, \xi)\right|_{\xi=t+2 x-2 u} d u\right), \quad m=1,2, \ldots, n .
\end{aligned}
$$

Отсюда в силу леммы и оценки (4) имеем

$$
\begin{aligned}
\left\|D_{t}^{1 / n} A\right\|(x) \leqslant & \sum_{k=0}^{n-1} \frac{2^{1-(k+1) / n}}{\Gamma(2-(k+1) / n)} \int_{x}^{\infty} u^{1-(k+1) / n}\left|q_{k}(u)\right| d u \cdot \exp \{\sigma(x)\} \\
\left\|\left(D_{t}^{1 / n}\right)^{m} A\right\|(x) \leqslant & \sum_{k=0}^{n-m} \frac{2^{1-(k+m) / n}}{\Gamma(2-(k+m) / n)} \int_{x}^{\infty} u^{1-(k+m) / n}\left|q_{k}(u)\right| d u \cdot \exp \{\sigma(x)\} \\
& +\sum_{k=1}^{m-1} \frac{2^{1-k / n}}{\Gamma(2-k / n)} \int_{x}^{\infty} u^{1-k / n}\left|q_{n-m+k}^{\prime}(u)\right| d u \\
& +\sum_{k=1}^{m-1} \int_{x}^{\infty}\left|q_{n-m+k}(u)\right| \cdot\left\|\left(D_{t}^{1 / n}\right)^{k} A\right\|(u) d u, \quad m=2,3, \ldots, n .
\end{aligned}
$$

Следовательно, производные $\left(D_{t}^{1 / n}\right)^{m} A(x, t), m=1,2, \ldots, n$, суммируемы по $t$ на интервале $(0, \infty)$.

3. Применяя оператор $I_{t}^{(n-1) / n}$ к обеим частям равенств $(14),(18)$ и используя оценки $(9)$ и $(4)$, непосредственно получаем, что функция $A(x, t)$ удовлетворяет условиям (6) и $(7)$.

4. Если функции $q_{0}(x), q_{1}^{\prime}(x), \ldots, q_{n-1}^{\prime}(x)$ непрерывно дифференцируемы и интегралы

$$
\int_{0}^{\infty}(1+u)\left|q_{0}^{\prime}(u)\right| d u, \quad \int_{0}^{\infty}(1+u)^{1-1 / n}\left|q_{k}^{\prime \prime}(u)\right| d u, \quad k=1,2, \ldots, n-1,
$$

сходятся, то, рассуждая так же, как на втором этапе, можно показать, что существуют суммируемые по $t$ производные $D_{x}^{2} A(x, t), D_{x}\left(D_{t}^{1 / n}\right)^{m} A(x, t), m=1,2, \ldots, n$, и

$$
\lim _{t \rightarrow \infty} I_{t}^{(n-1) / n}\left(D_{t}^{1 / n}\right)^{m} D_{x} A(x, t)=0 .
$$


Для того чтобы функция вида (3) удовлетворяла уравнению (1), должно вьполняться равенство

$$
\begin{aligned}
\int_{0}^{\infty} & D_{x}^{2} A(x, t) \cdot \exp \left\{-\lambda^{n} t\right\} d t-2 \lambda^{n} \int_{0}^{\infty} D_{x} A(x, t) \cdot \exp \left\{-\lambda^{n} t\right\} d t \\
& -\sum_{k=1}^{n-1} \lambda^{k} q_{k}(x)\left(1+\int_{0}^{\infty} A(x, t) \exp \left\{-\lambda^{n} t\right\} d t\right)=0 .
\end{aligned}
$$

С другой стороны, используя формулу (12) при $k=1$ и соотношения (7), интегрированием по частям получаем

$$
\begin{aligned}
\lambda \int_{0}^{\infty} A(x, t) \exp \left\{-\lambda^{n} t\right\} d t & =\lambda^{n} \int_{0}^{\infty} I_{t}^{(n-1) / n} A(x, t) \cdot \exp \left\{-\lambda^{n} t\right\} d t \\
& =\left.I_{t}^{(n-1) / n} A(x, t)\right|_{t=0}+\int_{0}^{\infty} D_{t}^{1 / n} A(x, t) \cdot \exp \left\{-\lambda^{n} t\right\} d t
\end{aligned}
$$

Аналогично, имеем

$$
\begin{aligned}
\lambda^{k} \int_{0}^{\infty} A(x, t) \exp \left\{-\lambda^{n} t\right\} d t= & \left.\sum_{m=0}^{k-1} \lambda^{m} I_{t}^{(n-1) / n}\left(D_{t}^{1 / n}\right)^{m} A(x, t)\right|_{t=0} \\
& +\int_{0}^{\infty}\left(D_{t}^{1 / n}\right)^{k} A(x, t) \cdot \exp \left\{-\lambda^{n} t\right\} d t, \\
& k=1,2, \ldots, n-1, \\
\lambda^{n} \int_{0}^{\infty} D_{x} A(x, t) \cdot \exp \left\{-\lambda^{n} t\right\} d t= & \left.\sum_{m=0}^{n-1} \lambda^{m} I_{t}^{(n-1) / n}\left(D_{t}^{1 / n}\right)^{m} D_{x} A(x, t)\right|_{t=0} \\
& +\int_{0}^{\infty}\left(D_{t}^{1 / n}\right)^{n} D_{x} A(x, t) \cdot \exp \left\{-\lambda^{n} t\right\} d t .
\end{aligned}
$$

Подставим эти выражения в (19) и проведем некоторые упрощения, придем к соотношению

$$
\begin{aligned}
\int_{0}^{\infty} & \left(D_{x}^{2} A(x, t)-2\left(D_{t}^{1 / n}\right)^{n} D_{x} A(x, t)-\sum_{m=0}^{n-1} q_{m}(x)\left(D_{t}^{1 / n}\right)^{m} A(x, t)\right) \exp \left\{-\lambda^{n} t\right\} d t \\
- & \lambda^{n-1}\left(\left.2 I_{t}^{(n-1) / n} D_{x} A(x, t)\right|_{t=0}+q_{n-1}(x)\right)-\lambda^{n-2}\left(\left.2 I_{t}^{(n-1) / n} D_{t}^{1 / n} D_{x} A(x, t)\right|_{t=0}\right. \\
& \left.+\left.q_{n-1}(x) I_{t}^{(n-1) / n} A(x, t)\right|_{t=0}+q_{n-2}(x)\right)-\cdots \\
& -\left(\left.2 I_{t}^{(n-1) / n}\left(D_{t}^{1 / n}\right)^{n-1} D_{x} A(x, t)\right|_{t=0}\right. \\
& \left.+\left.q_{n-1}(x) I_{t}^{(n-1) / n}\left(D_{t}^{1 / n}\right)^{n-2} A(x, t)\right|_{t=0}+\cdots+q_{0}(x)\right)=0 .
\end{aligned}
$$

Так как функция $A(x, t)$ удовлетворяет условию $(6)$, внеинтегральные члены в последнем равенстве обрашаются в 0 , следовательно, она будет удовлетворять и уравнению (8). Теорема доказана. 
ЗАмЕчАниЕ 1. На каждом секторе

$$
\Delta_{k}=\left\{\lambda:-\frac{\pi}{2 n}+\frac{k \pi}{n} \leqslant \arg \lambda \leqslant \frac{\pi}{2 n}+\frac{k \pi}{n}\right\}, \quad k=1,2, \ldots, 2 n,
$$

существует решение $f_{k}(x, \lambda)$ уравнения $(1)$, удовлетворяющее условию

$$
\lim _{x \rightarrow \infty} f_{k}(x, \lambda) \exp \left\{(-1)^{k} \lambda^{n} x\right\}=1
$$

и представимое в виде

$$
f_{k}(x, \lambda)=\exp \left\{(-1)^{k-1} \lambda^{n} x\right\}+\int_{x}^{\infty} A(x, t-x) \exp \left\{(-1)^{k-1} \lambda^{n} t\right\} d t .
$$

Используя свойства ядер $A_{l}(x, t)$, можно исследовать прямую и обратную задачи рассеяния для уравнения (1) с граничным условием $y(0)=0$. Этому вопросу будет посвящена одна из следующих работ автора.

ЗАмЕчАнИЕ 2. Используя формулу (12), нетрудно показать, что при $\lambda \in \Delta_{0}, t-x \geqslant 0$ имеет место оценка

$$
\left|\frac{1-\exp \left\{-2 \lambda^{n}(t-x)\right\}}{2 \lambda^{n-k}}\right| \leqslant \frac{2^{1-k / n}}{\Gamma(2-k / n)}(t-x)^{1-k / n}, \quad k=0,1, \ldots, n-1 .
$$

Тогда из уравнения (10) стандартньм методом (см., например, [8], [9]) получаем

$$
\left|f(x, \lambda) \exp \left\{\lambda^{n} x\right\}-1\right| \leqslant \frac{C \sigma(x)}{1+|\lambda|}, \quad \lambda \in \Delta_{0}, \quad C>0 .
$$

Отсюда согласно теореме типа Винера-Пэли $[6$, с. 443$]$ существует функция $B(x, t)$ такая, что

$$
f(x, \lambda)=\exp \left\{-\lambda^{n} x\right\}\left(1+\int_{0}^{\infty} B(x, t) e_{\rho}(t ; \lambda) d t\right),
$$

где $e_{\rho}(t ; \lambda)=t^{1 / \rho-1} E_{\rho}\left(-\lambda t^{1 / \rho} ; 1 / \rho\right), \rho=n /(2 n-1)$, а $E_{\rho}(z ; \mu)$ - функция Миттага-Леффлера. Как и в [9], можно изучать свойства функции $B(x, t)$.

ЗАМЕЧАНИЕ 3. Если вьполняются условия а), б) и

$$
\int_{0}^{\infty}\left|q_{n}(t)\right| d t<+\infty, \quad \int_{0}^{\infty}(1+t)\left|q_{n}^{\prime}(t)\right| d t<+\infty
$$

то для решения $f(x, \lambda)$ уравнения

$$
y^{\prime \prime}-\sum_{k=0}^{n} q_{k}(x) y=\lambda^{2 n} y
$$

удовлетворяющего условию (2), имеет место следующее треугольное представление:

$$
f(x, \lambda)=\exp \left\{-\lambda^{n} x+\frac{1}{2} \int_{x}^{\infty} q_{n}(t) d t\right\}+\int_{0}^{\infty} A(x, t-x) \exp \left\{-\lambda^{n} t\right\} d t .
$$

Отметим, что для $n=1$ это представление получено в работе [10] (см. также [8, с. 186]). 


\section{СПИСОК ЦИТИРОВАННОЙ ЛИТЕРАТУРЫ}

[1] Левитан Б. М. Теория операторов обобщенного сдвига. М.: Наука, 1973.

[2] Левитан Б. М. Обратные задачи Штурма-Лиувилля. М.: Наука, 1984.

[3] Левин Б.Я.Преобразования типа Фурье и Лапласа при помощи решений дифференциального уравнения второго порядка // Докл. АН СССР. 1956. Т. 106. № 2. С. 187-190.

[4] Марченко В. А. Операторы Штурма-Лиувилля и их приложения. Киев: Наукова думка, 1977.

[5] Самко С.Г., Килбас А. А., Маричев О. И. Интегралы и производные дробного порядка и некоторые их приложения. Минск: Наука и техника, 1987.

[6] Джрбашян М. М. Интегральные преобразования и представления функций в комплексной области. М.: Наука, 1966.

[7] Сидоров Ю. В., Федорюк М. В., Шабунин М. И. Лекции по теории функций комплексного переменного. М.: Наука, 1982.

[8] Шадан К., Сабатье П. Обратные задачи в квантовой теории рассеяния. М.: Мир, 1980.

[9] Гусейнов И. М. О некоторых представлениях для решения дифференциальных уравнений высокого порядка // Изв. АН АзССР. Сер. физ.-техн. и матем. наук. 1986. № 1. С. 27-32.

[10] Jaulent M., Jean C. The inverse $s$-wave scattering problem for a class of potentials depending on energy // Comm. Math. Phys. 1972. V. 28. P. 117-220.

Бакинский государственный университет

Поступило

им. М.А. Расулзаде

15.08 .95 